\title{
High tie versus low tie in rectal surgery: comparison of anastomotic perfusion
}

\author{
Niels Komen • Juliette Slieker • Peter de Kort • J. H. W. de Wilt • Erwin van der Harst • \\ Peter-Paul Coene $\cdot$ Martijn Gosselink $\cdot$ Geert Tetteroo $\cdot$ Eelco de Graaf \\ Ton van Beek • Rene den Toom • Wouter van Bockel • Cees Verhoef • Johan F. Lange
}

Accepted: 3 March 2011 / Published online: 29 March 2011

(C) The Author(s) 2011. This article is published with open access at Springerlink.com

\begin{abstract}
Purpose Both "high tie" (HT) and "low tie" (LT) are wellknown strategies in rectal surgery. The aim of this study was to compare colonic perfusion after HT to colonic perfusion after LT.

Methods Patients undergoing rectal resection for malignancy were included. Colonic perfusion was measured with laser Doppler flowmetry, immediately after laparotomy on the antimesenterial side of the colon segment that was to become the afferent loop (measurement A). This measurement was repeated after rectal resection (measurement B). The blood flow ratios (B/A) were compared between the HT group and the LT group.

Results Blood flow was measured in 33 patients, 16 undergoing HT and 17 undergoing LT. Colonic blood flow slightly decreased in the HT group whereas the flow
\end{abstract}

N. Komen $(\bowtie) \cdot J$. Slieker • P. de Kort $\cdot$ J. H. W. de Wilt • W. van Bockel $\cdot$ C. Verhoef $\cdot$ J. F. Lange

Department of Surgery, University Medical Center Rotterdam, Erasmus MC,

Rotterdam, The Netherlands

e-mail: nielskomen@hotmail.com

E. van der Harst • P.-P. Coene · M. Gosselink Department of Surgery, Maasstad Ziekenhuis,

Rotterdam, The Netherlands

G. Tetteroo $\cdot$ E. de Graaf

Department of Surgery, IJsselland Ziekenhuis,

Capelle aan den IJssel, The Netherlands

T. van Beek $\cdot$ R. den Toom

Department of Surgery, Ruwaard van Putten Ziekenhuis,

Spijkenisse, The Netherlands

J. H. W. de Wilt

Radboud University Nijmegen Medical Center,

Department of Surgery,

Nijmegen, The Netherlands increased in the LT group. The blood flow ratio was significantly higher in the LT group ( 1.48 vs. $0.91 ; p=0.04)$, independent of the blood pressure.

Conclusion This study shows the blood flow ratio to be higher in the LT group. This suggests that anastomoses may benefit from better perfusion when LT is performed.

Keywords High tie $\cdot$ Low tie $\cdot$ Perfusion $\cdot$ Colorectal surgery $\cdot$ Anastomosis

\section{Introduction}

To date, 100 years after the introduction of the low tie and high tie techniques for colorectal surgery by Miles and Moynihan, respectively [1,2], the discussion on which is the best technique continues, as illustrated by two recently published reviews [3, 4].

Titu et al. have summarized literature comparing low tie and high tie techniques for curative colorectal surgery [3]. They concluded that no undisputable evidence favouring one technique exists. Nevertheless, they propagate the high tie technique since it allows better lymph node retrieval and therefore a more accurate tumour staging.

In another review comparing the low tie with the high tie technique, Lange et al. distinguished three aspects in the discussion: oncological, anatomical and technical [4]. They concluded that for each aspect the evidence is insufficient to favour one technique. Nevertheless, they favour the low tie technique since it is less invasive, also with regard to colonic innervation and motility, and it would be beneficial for anastomotic perfusion compared to the high tie technique.

Adequate anastomotic perfusion is considered essential for anastomotic healing. Performing a high tie (HT) technique allows anastomotic perfusion only through the 
marginal artery, which may lead to a decrease in anastomotic perfusion $[5,6]$. When a low tie (LT) technique is performed, anastomotic perfusion is allowed not only through the marginal artery, but through the left colic artery and its ascending branch as well. This anatomical reality suggests that anastomotic perfusion is higher after low tie; however, no evidence exists supporting this hypothesis.

The aim of this study is to compare the high tie technique to the low tie technique with regard to anastomotic perfusion.

\section{Patients and methods}

Patients planned for elective rectal resection for malignancy in four participating hospitals, with nine participating surgeons, were eligible for this non-randomized, prospective study. The procedure was represented by a Total Mesorectal Excision with or without anastomosis.

Blood flow was measured with the $\mathrm{O} 2 \mathrm{C}$ system. The $\mathrm{O} 2 \mathrm{C}$ system ("oxygen to see", Lea Medizin Technik, Giessen, Germany) is a laser Doppler flowmetry system that has often been used to measure intestinal blood flow for research purposes [7, 8]. Blood flow, expressed in arbitrary units, is determined by analysing the Doppler frequency shifts in laser light $(820 \mathrm{~nm})$ reflected from moving red blood cells. The laser light is emitted into the tissue, and the backscattered light is detected with a flat probe with a measurement depth of 4-6 mm (Lea Medizin Technik, Giessen, Germany). The $\mathrm{O} 2 \mathrm{C}$ measurement frequency is $30 \mathrm{~Hz}$.

Measurements were performed at two moments during the operation, being (a) right after median laparotomy and (b) just before construction of the anastomosis or colostomy, in case of abdominoperineal resection. The measurements were performed on the antimesenterial, serosal side of the colon segment that was to become, or was after resection (at moment b), the proximal loop. For all measurements, after placement of the flat probe, the flow measurement was allowed to stabilize until a constant flow was measured. Afterwards, the flow was recorded for $30 \mathrm{~s}$, obtaining 15 values. The mean of these 15 measurements was used to calculate the blood flow ratio (BFR), B/A. During the measurements, the blood pressure was measured as well, and the mean arterial pressure (MAP) was calculated.

The high tie technique was defined as ligation of the inferior mesenteric artery (IMA) at its origin. The low tie technique was defined as ligation of the superior rectal artery (SRA), just below the branching of the left colic artery (Fig. 1). The surgeon decided which technique was used.

The BFR distribution was normalised by a logarithmic transformation and compared between the HT and LT groups by means of an unpaired $t$ test. MAP was compared between moments A and B with a paired sample $t$ test. Statistical analysis was performed with SPSS 15.0.

\section{Results}

During 1 year, 33 patients were included in four different medical centres. A HT was performed in 16 patients $(48 \%)$ of whom $12(75 \%)$ received a primary anastomosis. A LT was performed in 17 patients (52\%) of whom also 12 (71\%) received a primary anastomosis. In all patients receiving a primary anastomosis, the splenic flexure was mobilised. Nine patients $(75 \%)$ in the high tie group and ten patients $(83 \%)$ in the low tie group received a defunctioning stoma. The mean number of lymph nodes harvested in the high tie group was 11 (range, 6-23); in the low tie group, 12 (range,
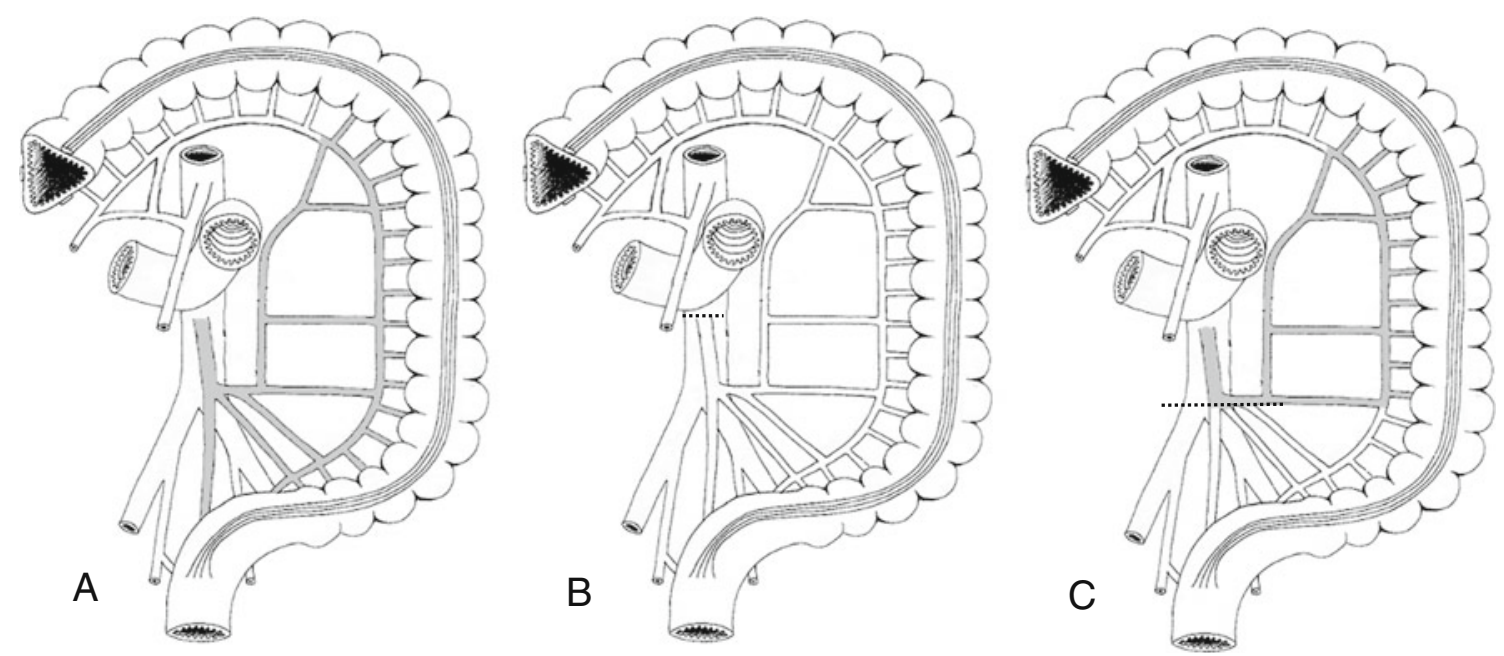

Fig. 1 The vasculature of the colon. a Grey indicates the flow area of IMA. b The dashed line indicates the level of ligature in HT, leaving no flow in the inferior mesenteric artery and its branches. $\mathbf{c}$ The dashed line indicates the level of ligature in LT; grey indicates the flow area of IMA after LT 
Table 1 Patient characteristics of included patients

\begin{tabular}{|c|c|c|c|}
\hline Baseline characteristics & High tie & Low tie & $p$ value \\
\hline Gender $(\mathrm{M} / \mathrm{F})$ & $11 / 5$ & $12 / 5$ & 1.000 \\
\hline Age (years) & $55 \pm 17$ & $61 \pm 13$ & 0.363 \\
\hline BMI $\left(\mathrm{kg} / \mathrm{m}^{2}\right)$ & $25 \pm 3$ & $27 \pm 7$ & 0.473 \\
\hline Operation & & & 1.000 \\
\hline APR & 4 & 5 & \\
\hline LAR & 12 & 12 & \\
\hline Neoadjuvant therapy & & & 0.024 \\
\hline RT & 14 & 8 & \\
\hline No RT & 2 & 9 & \\
\hline ASA score & & & 0.170 \\
\hline I & 7 & 5 & \\
\hline II & 8 & 6 & \\
\hline III & 1 & 6 & \\
\hline Cardiovascular comorbidity & $2(13 \%)$ & $4(24 \%)$ & 0.656 \\
\hline Operating time (minutes) & $160(100-340)$ & $145(45-225)$ & 0.450 \\
\hline Tumour stage & & & 0.250 \\
\hline 0 & 1 & 0 & \\
\hline I & 4 & 4 & \\
\hline IIa & 2 & 4 & \\
\hline IIb & 1 & 0 & \\
\hline IIIa & 2 & 3 & \\
\hline IIIb & 1 & 3 & \\
\hline IIIc & 0 & 1 & \\
\hline IV & 4 & 0 & \\
\hline
\end{tabular}

$M / F$ Male/Female, $B M I$ body mass index (kilograms per square meter), $A P R$ abdominoperineal resection, $L A R$ low anterior resection, $R T$ radiotherapy, ASA score American Society of Anaesthesiologists score

$6-33)(p=0.35)$. The mean number of positive lymph nodes harvested in the high tie group was $3(1-5)$; in the low tie group, 4 (1-9) $(p=0.32)$.

Two patients developed anastomotic leakage, one in the HT group and one in the LT group. In the HT group, significantly more patients received neoadjuvant radiotherapy. No significant differences were found in the remaining baseline characteristics (Table 1).

The mean BFR was significantly higher in the LT group as depicted in Table 2, whereas the blood pressure during measurements was not significantly different as depicted in Table 3.

Table 2 Comparison of blood flow ratios between the HT and LT techniques

\begin{tabular}{llccc}
\hline Ratio & HT/LT & Mean ratio & Std. error of the mean & $p$ value \\
\hline B/A & HT & 0.91 & 0.24 & 0.04 \\
& LT & 1.48 & 0.32 & \\
\hline
\end{tabular}

Table 3 MAP measured during the blood flow measurements at time points $\mathrm{A}$ and $\mathrm{B}$, respectively

\begin{tabular}{llccc}
\hline Group & MAP A/B & Mean & Std. error of the mean & $p$ value \\
\hline HT & A & 67.1 & 2.2 & 0.473 \\
& B & 64.2 & 3.2 & \\
LT & A & 69.8 & 4.4 & 0.075 \\
& B & 75.7 & 2.0 & \\
\hline
\end{tabular}

\section{Discussion}

To date, the discussion on the matter of high tie versus low tie continues. This study focuses on the colorectal vasculature and the flow change after HT or LT.

Seike et al. found the colonic blood flow to vastly decrease after ligation of IMA or SRA [5], with the subsequent conclusion that this could be an unavoidable factor in the pathophysiology of colorectal anastomotic leakage. However, this study shows otherwise. After a HT procedure, only a slightly decreased blood flow was observed at the end of the operation (BFR 0, 91), whereas an increased blood flow was measured after LT (BFR 1, 48). The blood flow changes occurred independently from the systemic blood pressure (Table 3). These different findings may be explained by the time interval between arterial ligation and measurement. Seike et al. performed their measurements immediately after clamping of the artery. In this study the first measurement was performed immediately after laparotomy, and the second measurement, just before construction of the anastomosis or colostomy, i.e. at the end of the operation. Therefore, the interval between ligation and measurement is much longer in this study compared to the aforementioned study. This suggests that over time, a recruitment of colonic arteries occurs, allowing recovery of blood flow. In order to study whether these blood flow changes are permanent or not, blood flow measurements in the postoperative period would be interesting. In addition, since anastomotic leakage is generally detected around the eighth postoperative day [9], it could provide important information on the pathophysiological processes concerning blood flow leading to AL.

The BFR was significantly higher after LT which means LT allows better perfusion of the proximal anastomotic loop at the end of the operation. Most likely, this is due to the preservation of the left colic artery and its ascending branch. In addition to the marginal artery, these arteries allow a second pathway for blood supply and faster and/or a more extensive recruitment of colonic arteries. Therefore, since good perfusion is essential for proper anastomotic healing, LT would be the preferred technique for this aspect of the high tie-low tie comparison. 
The average BFR after LT shows an increase in blood flow compared to the initial value at the end of the operation. This has been described before by Karlicek et al. [10] and could be due to reactive hyperaemia as a result of colon manipulation. However, it could also be due to a variety of ischaemia reperfusion injuries (IRI). These injuries have been well described in animal models in which an IRI leads to visible hyperaemia and decreased anastomotic strength [11]. This response is probably also present after HT; however, it is more outspoken after LT most likely due to preservation of the left colic artery. Whether these findings have an impact on the incidence of anastomotic leakage should be evaluated by analysing the blood flow during the postoperative period or in a similar but larger study.

The $\mathrm{O} 2 \mathrm{C}$ allows non-invasive measurement of blood flow; however, the measurements are sensitive to several variables. First, it depends on placement of the probe. The probe has to be placed on the exact same spot for perfect comparability. Since it is virtually impossible to mark a spot on the colon without influencing the local blood flow or without hindering the progress of the operation, placement of the probe will be slightly variable. Second, the measurements are sensitive to different pressures applied on the probe when holding it in the right position. Higher pressures are likely to lead to more compressed arteries and a lower blood flow. In order to limit the influence of these variables on the outcome, measurements were performed by the same surgeon, allowing reproducibility of the measurement. In addition, the blood flow ratio was calculated for which the first measurement served as a control.

The use of a ratio also allowed standardizing intrinsic, patient-related differences like microangiopathy due to atherosclerosis and diabetes mellitus.

Table 1 shows baseline characteristics to be comparable between HT and LT except for radiotherapy. Significantly more people in the HT group received neoadjuvant radiotherapy. This is, however, unlikely to have an effect on the blood flow in the proximal anastomotic loop since this loop is located outside the radiation field.

In addition, the high tie group contained a higher number of patients with metastasized disease (stage 4 present in $25 \%$ in the HT group vs. $0 \%$ in the low tie group). This difference most likely illustrates the participating surgeons having preferred to perform a high tie technique in patients with metastasized disease.

\section{Conclusion}

When comparing high tie ligation to low tie ligation, this study shows the perfusion of the proximal loop of the anastomosis to be better after low tie ligation. Considering neither of both techniques is favourable on the oncological or technical aspect, low tie ligation may be the technique of choice in patients undergoing rectum resection.

Conflict of interest The authors have no conflict of interest.

Open Access This article is distributed under the terms of the Creative Commons Attribution Noncommercial License which permits any noncommercial use, distribution, and reproduction in any medium, provided the original author(s) and source are credited.

\section{References}

1. Miles WE (1971) A method of performing abdomino-perineal excision for carcinoma of the rectum and of the terminal portion of the pelvic colon (1908). CA Cancer J Clin 21(6):361-364

2. Moynihan B (1908) The surgical treatment of cancer of the sigmoid flexure and rectum. Surg Gynecol Obstet 6:463-466

3. Titu LV, Tweedle E, Rooney PS (2008) High tie of the inferior mesenteric artery in curative surgery for left colonic and rectal cancers: a systematic review. Dig Surg 25(2):148-157

4. Lange $\mathrm{MM}$ et al (2008) Level of arterial ligation in rectal cancer surgery: low tie preferred over high tie. A review. Dis Colon Rectum 51(7):1139-1145

5. Seike K et al (2007) Laser Doppler assessment of the influence of division at the root of the inferior mesenteric artery on anastomotic blood flow in rectosigmoid cancer surgery. Int $\mathrm{J}$ Colorectal Dis 22(6):689-697

6. Dworkin MJ, Allen-Mersh TG (1996) Effect of inferior mesenteric artery ligation on blood flow in the marginal artery-dependent sigmoid colon. J Am Coll Surg 183(4):357-360

7. De Bruin AF et al (2009) The impact of chronic smoking on rectal mucosal blood flow. Tech Coloproctol 13(4):269-272

8. Albuszies G et al (2005) Effect of increased cardiac output on hepatic and intestinal microcirculatory blood flow, oxygenation, and metabolism in hyperdynamic murine septic shock. Crit Care Med 33(10):2332-2338

9. Komen N et al (2009) After-hours colorectal surgery: a risk factor for anastomotic leakage. Int J Colorectal Dis 24(7):789-795

10. Karliczek A et al (2010) Intraoperative assessment of microperfusion with visible light spectroscopy for prediction of anastomotic leakage in colorectal anastomoses. Colorectal Dis 12:1018-1025

11. Posma LA et al (2007) Transient profound mesenteric ischemia strongly affects the strength of intestinal anastomoses in the rat. Dis Colon Rectum 50(7):1070-1079 